\title{
Many-Body and Correlation Effects on Parametric Polariton Amplification in Semiconductor Microcavities
}

\author{
Salvatore Savasta, Omar Di Stefano, and Raffaello Girlanda \\ Istituto Nazionale per la Fisica della Materia INFM and Dipartimento di Fisica della Materia e Tecnologie Fisiche Avanzate, \\ Universit à di Messina, Salita Sperone 31, I-98166 Messina, Italy
}

\begin{abstract}
The complexity induced by the Coulomb interaction between electrons determines the noninstantaneous character of exciton-exciton collisions. We show that the exciton-photon coupling in semiconductor microcavities is able to alter the exciton dynamics during collisions strongly affecting the effective scattering rates. Our analysis clarifies the origin of the great enhancement of parametric gain observed when increasing the polariton splitting. It also demonstrates that exciton-exciton collisions in semiconductors can be controlled and engineered to produce almost decoherence-free collisions for the realization of all-optical microscopic devices.
\end{abstract}

DOI: 10.1103

The phase-coherent amplification of light-matter waves (cavity polaritons) recently observed is one of the most exciting developments in the field of semiconductor nonlinear optics [1-7]. It operates with analogous principles (but under very different conditions) of matterwave amplifiers based on ultracold atoms [8,9]. Very recently it has been shown that a semiconductor microcavity (SMC) can amplify (via phase-coherent amplification of polaritons) a weak light pulse more than 5000 times [7].

Cavity polaritons are two-dimensional eigenstates of SMCs which result from the strong resonant coupling between cavity-photon modes and two-dimensional excitons in embedded quantum wells (QWs) [10]. The dynamics and hence the resulting energy bands of these mixed quasiparticles are highly distorted with respect to those of bare excitons and cavity photons (Fig. 1). The exciton-photon coupling rate $V$ determines the splitting $(2 \mathrm{~V})$ between the two polariton energy bands. Coherent amplification of polaritons requires a coupling mechanism able to transfer polaritons from a reservoir (in this case provided by polaritons resonantly excited by a pump laser pulse on the lower polariton dispersion) to the signal mode, while conserving energy and momentum. Polaritons of different modes are coupled via their excitonic content, the coupling being provided mainly by the Coulomb interaction between excitons (also the anharmonic part of the exciton-photon interaction contributes). The microscopic theory of parametric polariton amplification [11] shows that the resulting Coulomb coupling strength is given by the exciton-exciton $(X X)$ scattering rate [12] $V_{X X} \simeq 1.52 E_{b} a_{0}^{2}\left(E_{b}\right.$ is the exciton binding energy and $a_{0}$ is the exciton Bohr radius) times the exciton fraction of the interacting polariton modes. This effective polariton-polariton interaction scatters a pair of pump polaritons into the lowest-energy state and into a higher-energy state (usually known as the idler mode). Energy and momentum conservation requires $2 \omega_{k}=$ $\omega_{0}+\omega_{2 k}$, where $\omega_{k}$ is the energy of a pump polariton injected with an in-plane wave vector $\mathbf{k}$ as shown in Fig. 1. The scattering process is stimulated by a weak signal beam injected perpendicular to the cavity $(\mathbf{k}=0)$ that is thus greatly amplified. It has recently been shown that increasing the exciton-photon coupling $V$ by inserting a large number of QWs into the cavity $(V=$ $V_{1} \sqrt{N_{\text {eff }}}$, where $V_{1}$ is the exciton-photon coupling for

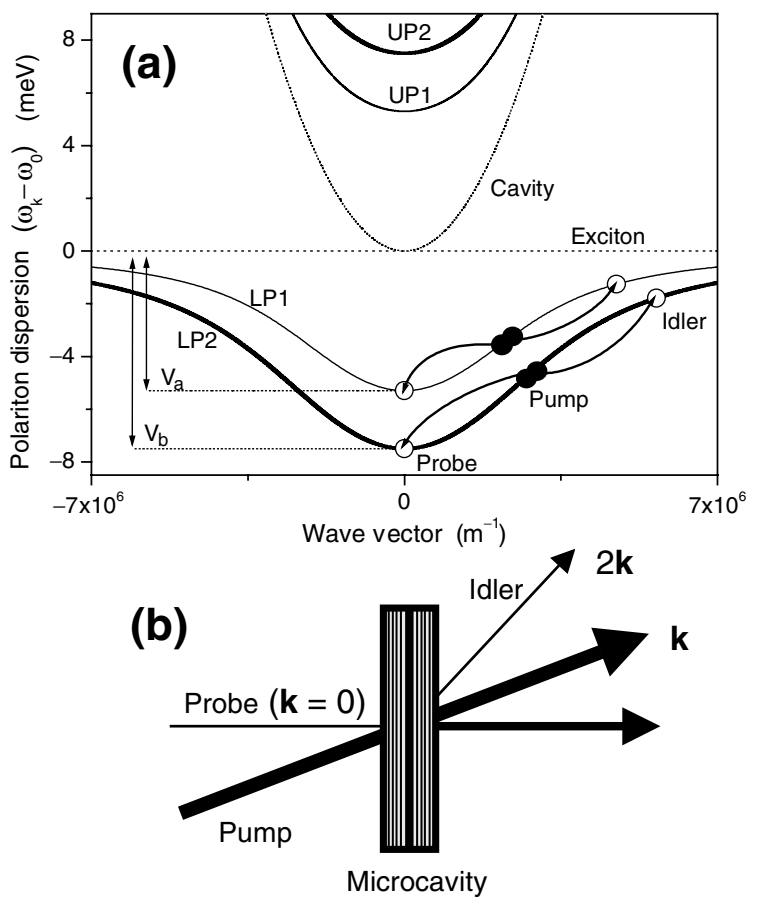

FIG. 1. Sketch of the polariton energy versus the in-plane wave vector (a), for two different exciton-photon coupling $\left(2 V_{a}=10.4\right.$ and $\left.2 V_{b}=15 \mathrm{meV}\right)$. UP and LP denote the splitted upper polariton and lower polariton curves. The parametric polariton-polariton scattering process is also depicted. (b) Schematic of the experiment. 
1 QW and the effective number of QWs $N_{\text {eff }}$ depends on the number of wells inside the cavity and their spatial overlap with the cavity mode), greatly increases amplification [7]. This spectacular enhancement of gain contrasts with the results of present theories [11,13] describing the process. According to mean-field descriptions, the gain is limited only by the pump depletion and can thus reach very high values (orders of magnitude higher than experimental findings, as shown in this Letter) also for small values of $V$. Moreover, according to these descriptions, inserting more QWs just lowers the polariton-polariton interaction, without changing the structure of pertinent equations. In particular we observe that the $X X$ interaction in the presence of more QWs results to be $V_{X X} / N_{\text {eff }}$, while the exciton and photon contents of the three interacting modes result in being independent of $V$. From this observation and from a direct inspection of the pertinent equations, it descends that, increasing the exciton-photon coupling, should not increase the amplification of a weak signal beam in contrast with the spectacular experimental observations [7].

Here we show that the great enhancement of gain, observed when increasing the polariton splitting, has its origins from the unique interplay of the noninstantaneous nature of $X X$ interactions [14] with the strong coupling regime giving rise to polaritons. The mean-field theory of the optical response of electron-hole pairs assumes that collisions are instantaneous and only the mean free time between collisions matters. Although the mean-field theory has enjoyed considerable success $[11,14]$, recent studies revealed that collisions between excitons are more complex (see [14] and references cited therein). They involve multiparticle (at least fourparticle) entangled states. This complexity, induced by the Coulomb interaction between electrons, determines the finite duration of collisions [12,14-18]. We find that the strong coupling of excitons with cavity photons, giving rise to polaritons, alters the excitonic dynamics during $X X$ collisions, producing a modification of the effective scattering rates. As a consequence, a variation of the exciton-photon coupling rate $V$ can affect significantly $X X$ collisions and hence the amplification process. To analyze the influence of the finite duration of $X X$ interactions on the parametric polariton amplification, we use a microscopic description where the hierarchy of higher order density matrices has been closed by invoking the dynamics controlled truncation (DCT) scheme [19]. The calculations have been performed in the so-called coherent limit. Furthermore, we included multiple scattering simply by replacing in the nonlinear sources, linear light, and polarization fields with the total fields. The intracavity light field is described within the quasimode scheme [16]. The time evolution of the coupled exciton $\left(P_{\mathbf{k}}\right)$ and photon waves $\left(E_{\mathbf{k}}\right)$ including finite duration of $X X$ collisions $[16,17]$ can be described by the following set of coupled equations,

$$
\begin{aligned}
\frac{\partial}{\partial t} E_{\mathbf{k}} & =-\left(\gamma_{c}+i \omega_{k}^{c}\right) E_{\mathbf{k}}+i V P_{\mathbf{k}}+t_{c} E_{\mathbf{k}}^{\mathrm{in}}, \\
\frac{\partial}{\partial t} P_{\mathbf{k}} & =-\left(\gamma_{x}+i \omega_{x}\right) P_{\mathbf{k}}+i V E_{\mathbf{k}}-i \Omega_{\mathbf{k}}^{\mathrm{NL}},
\end{aligned}
$$

where $\omega_{k}^{c}, \omega_{x}$, and $\gamma_{c}, \gamma_{x}$ are the energies and dephasing rates of cavity photons and QWs excitons, respectively. $E_{\mathbf{k}}^{\text {in }}$ describes input light pulses, $t_{c}$ determines the beam fraction passing the cavity mirror $\left(t_{c}=\sqrt{\gamma_{c}}\right.$ for a cavity with equal mirrors). The intracavity and the exciton field of a given mode $\mathbf{k}$ are coupled by $V$. The relevant nonlinear source term, able to couple waves with different inplane wave vector $\mathbf{k}$ [20], is given by $\Omega_{\mathbf{k}}^{\mathrm{NL}}=\left(\Omega_{\mathbf{k}}^{\text {sat }}+\right.$ $\left.\Omega_{\mathbf{k}}^{X X}\right) / N_{\text {eff }}$, where the first term originates from the phase-space filling of the exciton transition,

$$
\Omega_{\mathbf{k}}^{\text {sat }}=\frac{V}{n_{\text {sat }}} \sum_{\mathbf{k}^{\prime} \mathbf{k}^{\prime \prime}} P_{\mathbf{q}}^{*} P_{\mathbf{k}^{\prime \prime}} E_{\mathbf{k}^{\prime}},
$$

being $n_{\text {sat }}=7 /\left(4 \pi a_{0}^{2}\right)$ the exciton saturation density $\left(\mathbf{k}^{\prime}\right.$, $\mathbf{k}^{\prime \prime}$, and qare tied by the momentum conservation relation $\mathbf{k}+\mathbf{q}=\mathbf{k}^{\prime}+\mathbf{k}^{\prime \prime}$ ). $\Omega_{\mathbf{k}}^{X X}$ is the Coulomb interaction term. It dominates the coherent $X X$ coupling and for cocircularly polarized waves can be written as

$$
\begin{aligned}
\Omega_{\mathbf{k}}^{X X}=\sum_{\mathbf{k}^{\prime} \mathbf{k}^{\prime \prime}}[ & V_{X X} P_{\mathbf{q}}^{*}(t) P_{\mathbf{k}^{\prime \prime}}(t) P_{\mathbf{k}^{\prime}}(t) \\
& \left.\quad-i P_{\mathbf{q}}^{*}(t) \int_{-\infty}^{t} F\left(t-t^{\prime}\right) P_{\mathbf{k}^{\prime \prime}}\left(t^{\prime}\right) P_{\mathbf{k}^{\prime}}\left(t^{\prime}\right)\right] .
\end{aligned}
$$

$\Omega_{\mathbf{k}}^{X X}$ includes the instantaneous mean-field $X X$ interaction term $\left(V_{X X}\right)$ plus a noninstantaneous term originating from four-particle correlations. This coherent memory can be interpreted as a non-Markovian process involving the 2-particle (excitons) polarization waves interacting with a bath of four-particle correlations $[14,15]$. The analytical expression of $F(\tau)$ in terms of the two-exciton wave functions can be found in Refs. [12,16,18,19]. We observe that the strong exciton-photon coupling does not modify the memory kernel $F(\tau)$ as a consequence of the fact that four-particle correlations do not couple to cavity photons [16-18]. However, cavity effects are able to alter the phase dynamics of two-particle polarization waves $P_{\mathbf{k}}$ during collisions, i.e., on a time scale shorter than the decay time of the memory kernel $F(\tau)$. In particular, the phase of 2-particle polarization waves $P_{\mathbf{k}}$ in SMCs oscillates with a frequency $\omega_{k}$ (fixed by the polariton dispersion relations) modified respect to that of excitons in bare QWs. This fact produces a modification of the integral in Eq. (3). In this way the exciton-photon coupling $V$ affects the $X X$ collisions that govern the polariton amplification process. This mechanism can be more clearly understood simplifying the integral in Eq. (3). Let us consider a situation where the pump, the signal, and idler energies are all close to the corresponding polariton resonance values and the broadening is small compared to the polariton splitting $2 \mathrm{~V}$. Then it is a good approximation to replace the integral in Eq. (3) with a simpler expression [21], adopting the Weisskopf-Wigner approximation used 
to analyze the spontaneous emission associated with atomic transitions. Within this approximation, the dominant $X X$ interaction term for the signal (0) and idler (2k) modes can be written as [17]

$$
\begin{aligned}
\Omega_{0(2 \mathbf{k})}^{X X}= & \mathcal{F}\left(\omega_{0(2 \mathbf{k})}+\omega_{\mathbf{k}}\right)\left|P_{\mathbf{k}}(t)\right|^{2} P_{0(2 \mathbf{k})}(t) \\
& +\mathcal{F}\left(2 \omega_{\mathbf{k}}\right) P_{2 \mathbf{k}(0)}^{*}(t) P_{\mathbf{k}}^{2}(t),
\end{aligned}
$$

where the complex quantity $\mathcal{F}(\omega)$ is the Fourier transform of the memory kernel $F(\tau)$ plus the mean-field contribution $V_{X X}$. The first term produces a blueshift of the polariton resonance and introduces an intensitydependent dephasing mechanism. The second term provides the coupling mechanism able to transfer polaritons from the pump to the signal and idler modes. An analogous expression can be derived for the $X X$ interaction of the pump mode. This Weisskopf-Wigner description of four-particle correlation effects in SMCs sets out the relevance of polariton pairs in the scattering process. The energy of these pairs determines the effective scattering rates as shown by Eq. (4). The spectral function $\mathcal{F}(\omega)$ can be calculated by numerical diagonalization of the semiconductor Hamiltonian in the four-particle subspace. We calculated it for QW excitons following a recent microscopic approach [12,18] based on the T matrix (Fig. 2). The obtained four-particle spectral density displays strong variations within the spectral region of interest around $2 \omega_{0}$. In particular, moving towards the low energy region, the dispersive part $\operatorname{Re}(F)$ increases while the absorptive part $\operatorname{Im}(F)$ (it reflects the density of states of 2-exciton correlations [15]) that contrasts gain goes to zero. We observe that the energies of the pump, signal, and idler polaritons lowers with the increase of polariton splitting (2V) [see Fig. 1(a)]. The increase of $V$ thus modi-

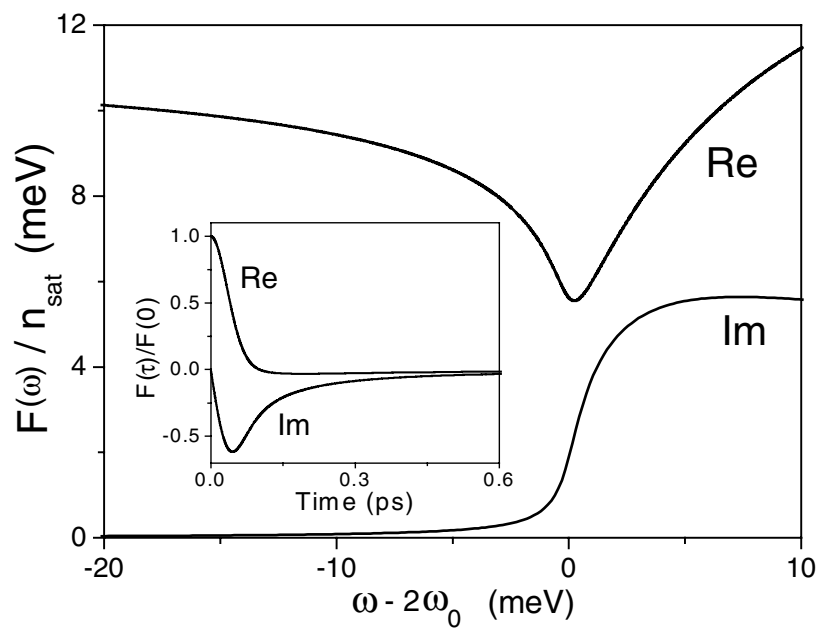

FIG. 2. Energy dependence of the effective exciton-exciton scattering potential. It has been calculated for a GaAs QW $7 \mathrm{~nm}$ wide with exciton binding energy $13.5 \mathrm{meV}$ and using as dephasing rate of the four-particle states $\Gamma=2 \gamma_{x}=0.58 \mathrm{meV}$. The tail of $\operatorname{Im}[\mathcal{F}(\omega)]$ at negative detuning $\left(\omega<2 \omega_{0}\right)$ is produced by $\Gamma$ and vanishes for $\Gamma \rightarrow 0$. The inset shows the dynamics of the memory kernel $F(\tau)$. fies the effective collision rates in such a way that favors the amplification process. This analysis explains the large increase of gain observed when maximizing the excitonphoton coupling [7]. The gain curve versus the input pump power, obtained solving numerically the system of integro-differential equations (1) for GaAlAs-based samples with splitting $2 V_{a}=10.6 \mathrm{meV}\left(N_{\text {eff }}=4\right)$ and $2 V_{b}=15 \mathrm{meV}\left(N_{\text {eff }}=8\right)$ [Fig. 3(a)], fully confirms this analysis. The calculated gain is defined as the total light intensity transmitted in the signal direction divided by the intensity transmitted in the absence of the pump beam [23]. The theoretical scheme here proposed permits a full quantitative analysis of gain versus the input pump power. An increase of less then a factor of $1 / 3$ in the polariton splitting produces an increase of the maximum achievable gain at $T=10 \mathrm{~K}$ [Fig. 3(a)] of more than 1 order of magnitude [Fig. 3(a)] in close agreement with experimental results [7]. The power dependence of gain (Fig. 3) shows an almost exponential growth and then saturates
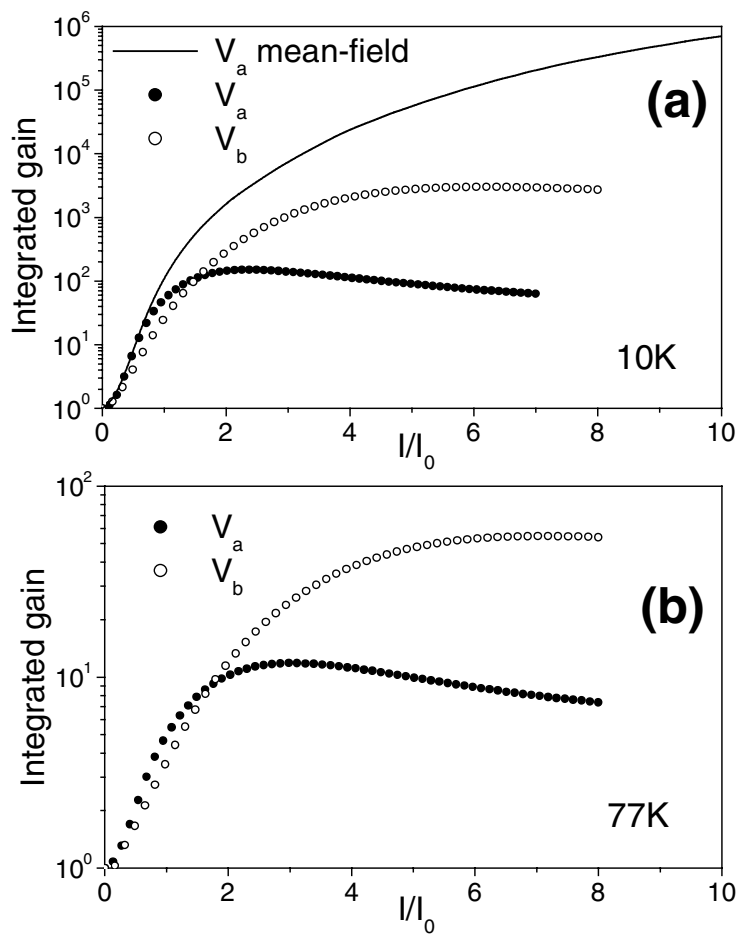

FIG. 3. Power dependence of the integrated gain calculated for two GaAlAs based samples with splitting $2 V_{a}=10.6 \mathrm{meV}$ and $2 V_{b}=15 \mathrm{meV}$. $I_{0}=10^{13}$ photons per $\mathrm{cm}^{2}$ per pulse, corresponding to 105 photons in a Bohr disk $\left(\pi a_{0}^{2}\right)$ per pulse. The exciting light pulses have 250 fs duration. Material parameters of the two samples are coincident except for $N_{\text {eff }}$. The decay rate of cavity photons through the mirrors $\gamma_{c}=$ $0.25 \mathrm{meV}$ is that of GaAlAs structures considered in Ref. [7]. The $7 \mathrm{~nm}$ wide QWs have a binding energy $E_{b}=13.5 \mathrm{~nm}$. Exciton homogeneous broadenings have been extracted from polariton linewidth measurements [22], $2 \gamma_{x}=0.6 \mathrm{meV}$ at $T=$ $10 \mathrm{~K}(\mathrm{a})$, and $2 \gamma_{x}=1.4 \mathrm{meV}$ at $T=77 \mathrm{~K}$ (b). The gain curves have been obtained fully optimizing incidence angles, central frequencies of the input light beams and cavity mode energy. 


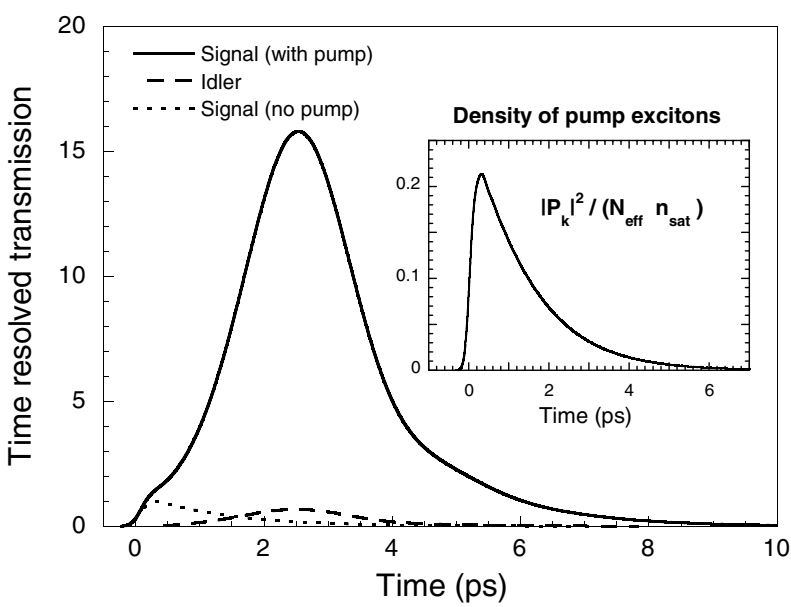

FIG. 4. Time-resolved transmission of the probe (with and without the presence of the pump beam) and additional emission (appearing in the presence of the pump beam) along the direction of the idler mode $\left(\theta \approx 47^{\circ}\right)$, showing the ultrafast dynamics of the parametric amplification. The sample is $V_{b}$, the temperature is $10 \mathrm{~K}$, and the pump intensity is $I=I_{0}$. The inset displays the time behavior of the pump-exciton density.

at high powers. The saturation of gain is mainly determined by the nonlinear absorption that is most relevant for the idler beam [according to Eq. (4) the idler nonlinear absorption is determined by $\left.\operatorname{Im} \mathcal{F}\left(\omega_{2 \mathbf{k}}+\omega_{\mathbf{k}}\right)\right]$. We observe that the increase of the idler nonlinear absorbance is highly superlinear because the increase of the pump power produces both a direct increase of the exciton density and an increase of $\operatorname{Im} \mathcal{F}\left(\omega_{2 \mathbf{k}}+\omega_{\mathbf{k}}\right)$ as a consequence of the blueshift of the polariton-pair resonance $\omega_{2 \mathbf{k}}+\omega_{\mathbf{k}}$ induced by $\operatorname{Re} \mathcal{F}$. We observe that mean-field calculations [also reported in Fig. 3(a)] overestimate the experimentally observed gain of more than 4 orders of magnitude, neither are able to reproduce the strong dependence of gain on the exciton-photon coupling $V$, that is a direct consequence of the inner dynamics of $X X$ collisions. Such a dramatic failure of the mean-field theory (at pretty high densities) is surprising and clearly shows the technological relevance that many-body correlations and their optical control may have in the future development of semiconductor all-optical devices. Figure 3(b) displaying results for $T=77 \mathrm{~K}$, shows how the increase of the exciton-photon coupling favours hightemperature operation. At $T=77 \mathrm{~K}$ the sample $V_{a}$ is still able to sustain significant gain about 6 times larger than that of sample $V_{b}$. The apparent discrepancy of the threshold values reported in Fig. 3 with the corresponding measurements [7] is due to a different definition of gain. Calculating the threshold values according to Ref. [7], we obtain $I=0.8 I_{0}\left(V_{a}\right)$ and $I=0.96 I_{0}\left(V_{b}\right)$ at $T=77 \mathrm{~K}$, corresponding to a density of pump excitons $\max \left[\left|P_{k}\right|^{2}\right] /\left(N_{\text {eff }} n_{\text {sat }}\right) \simeq 00.18$.

The low-temperature time dynamics of the parametric amplification is displayed in Fig. 4. The appearance of an additional light beam in a direction allowing momentum conservation (idler mode), also observed experimentally [1] confirms the coherent nature of this scattering process involving pairs of polariton waves. The smallness of emission in the idler direction (as compared to the amplified signal) is a consequence of the low photon content of polaritons at high angles.

The results here presented explain why the polariton parametric amplifier works despite in exciton systems losses increase with the excitation density owing to decoherence produced by collisions between excitons. The results here shown demonstrate that exciton-exciton collisions in semiconductors can be controlled and engineered. Moreover, they give precise indications to produce almost decoherence-free collisions for the realization of all-optical microscopic switches and amplifiers. The availability of decoherence-free collisions appears crucial for the quantum control and manipulation of the polariton wave function inside the cavity and for the realization of microscopic sources of nonclassical light.

The work benefited from discussions with J. J. Baumberg, G. Bongiovanni, C. Ciuti, A Quattropani, M. Saba, and P. Schwendimann. We also thank G. Bongiovanni, N. H. Kwong, M. Saba, and R. Takayama for providing unpublished results.

[1] P. G. Savvidis et al., Phys. Rev. Lett. 84, 1547 (2000).

[2] G. Dasbach et al., Phys. Rev. B 62, 13076 (2000).

[3] R. M. Stevenson et al., Phys. Rev. Lett. 85, 3680 (2000).

[4] P. G. Savvidis et al., Phys. Rev. B 62, R13 278 (2000).

[5] J. J. Baumberg et al., Phys. Rev. B 62, R16 247 (2000).

[6] G. Messin et al., Phys. Rev. Lett. 87, 127403 (2001).

[7] M. Saba et al., Nature (London) 414, 731 (2002).

[8] S. Inouye et al., Nature (London) 402, 641 (1999).

[9] M. Kozuma et al., Science 286, 2309 (1999).

[10] C. Weisbuch et al., Phys. Rev. Lett. 69, 3314 (1992).

[11] C. Ciuti et al., Phys. Rev. B 62, R4825 (2000).

[12] R. Takayama et al., Eur. Phys. J. B 25, 445 (2002).

[13] D. M. Whittaker, Phys. Rev. B 63, 193305 (2001).

[14] D. S. Chemla and J. Shah, Nature (London) 411, 549 (2001).

[15] T. Östreich et al., Phys. Rev. Lett. 74, 4698 (1995).

[16] S. Savasta and R. Girlanda, Phys. Rev. Lett. 77, 4736 (1996).

[17] S. Savasta et al., Phys. Rev. B 64, 073306 (2001).

[18] N. H. Kwong et al., Phys. Rev. Lett. 87, 027402 (2001).

[19] V. M. Axt and S. Mukamel, Rev. Mod. Phys. 70, 145 (1998).

[20] We neglected the wave-vector dependence of the coefficients driving nonlinear interactions as in the meanfield theory [11].

[21] The time dependence of polarization waves inside the integral can be written as $P_{k}=s_{k}(t) \exp \left(i \omega_{k} t\right)$ with $s_{k}$ assumed to be a slowly varying amplitude as compared to the rapid decay time of the memory kernel.

[22] M. Saba (private communication).

[23] We observe that total gain is a better candidate than peak gain for comparison with experiments since it is less sensitive to disorder effects. 